\title{
Soret and Dufour effects on unsteady boundary layer flow and heat transfer in copper- water nanofluid over a shrinking sheet with partial slip and stability analysis
}

\begin{abstract}
The influences of partial slip, Soret and Dufour on unsteady boundary layer flow, heat and mass transfer over shrinking sheet in Copper-water nanofluid are investigated numerically using Tiwari and Das's model. The governing equations are transformed to ordinary differential equations using similarity transformation. The system of equations is solved by boundary value problem with fourth order accuracy (bvp4c) program in Matlan and the numerical results are presented graphically to show the behaviour of the flow. The findings show that the dual solutions exist for certain range of suction effect $(\mathrm{s}>0.58)$ with the presence of partial slip effect. Besides, Soret effect increases the heat transfer and declines the mass transfer at the surface while Dufour effect showed the opposite trend. Since the dual solutions are obtained, the stability analysis is performed and it is found that the first solution is a stable and physically-realizable solution.
\end{abstract}

Keyword: Copper-water nanofluid; Dufour effect; Partial-slip effect; Soret effect; Stability analysis; Unsteady boundary layer 